\title{
Modeling of Fragmentation of Melt Droplets Triggered by Boiling Effect*
}

\author{
Qian LIN**, Zhe WANG**and Xuewu CAO** \\ **School of Nuclear Science and Engineering, Shanghai Jiao Tong University \\ 800 Dongchuan Rd., Shanghai, 200240, China \\ E-mail: sjtulq@sjtu.edu.cn
}

\begin{abstract}
The thermal fragmentation process of melt droplets in an energetic fuel coolant interaction is investigated. Boling effect is considered to be the triggering event, during the transition of heat transfer mode from film boiling to nucleate boiling and coolant periodically contacting with the droplet surface. The vapor bubble around droplet becomes unstable and collapses toward the surface of the droplet which induces the fragmentation of melt droplets. The vapor bubble collapse is modeled by writing a momentum equation for vapor bubble dynamics, an energy equation for each region of the droplet, coolant vapor and liquid and linking each region by the appropriate boundary conditions. And then a thermal fragmentation model triggered by boiling effect is developed and verified. By using the developed model, the fragmented mass of the droplet triggered by boiling effect is calculated. The result shows that the fragmentation rate is larger than that given by hydrodynamic model.
\end{abstract}

Key words: Fuel Coolant Interaction, Fragmentation, Heat Transfer Mode, Boiling Effect, Vapor Bubble Collapse

\section{Introduction}

Fuel Coolant Interactions (FCIs) are the important phenomena during the Core Disruptive Accidents (CDAs) in LMFBRs or Core Melt Accidents (CMAs) in LWRs. The fragmentation process is a key factor to determine the ratio of heat transferred to power in FCIs, which estimated possible damage degree. The fragmentation mechanisms of melt droplets under different conditions have been studied for many years. Corradini reviewed the mechanisms and models and categorized the fragmentation mechanisms into two classes; hydrodynamic and thermal effects ${ }^{(1)}$. The hydrodynamic fragmentation occurs when the melt droplets are subjected to external surface forces sufficient to overcome the droplet surface tension. Several kinds of fragmentation mechanisms have been proposed for the hydrodynamic effects: Rayleigh-Taylor instability, Kelvin-Helmholtz instability and boundary layer stripping. In thermal mechanism, the internal forces break the droplet into parts (fragments). The thermal effects include boiling, evaporation, internal pressurization, surface solidification effects and so on.

When the melt droplets are released into coolant, due to the evaporation of the coolant, a vapor film is formed around the droplet, as shown in Nelson's experiment ${ }^{(2)}$. The behavior of the vapor film is related to the temperatures of the droplet and the coolant, ambient pressure and properties of the coolant and the droplet. The objective of this study is to develop a fragmentation model triggered by boiling effect, based on the modeling of vapor bubble collapse around the droplet.

*Received 2 Aug., 2007 (No. 07-0337) [DOI: 10.1299/jpes.2.845] 


\section{Nomenclature}

$t$ time, $\mathrm{s}$

$T$ temperature, $\mathrm{K}$

$T_{m c}$ center temperature of melt droplet, $\mathrm{K}$

$T_{m s}$ surface temperature of melt droplet, $\mathrm{K}$

$T_{c i}$ interfacial temperature of vapor and coolant, $\mathrm{K}$

$T_{c 0}$ boundary temperature of coolant, $\mathrm{K}$

$T_{\text {sat }}$ saturated temperature, $\mathrm{K}$

$P$ pressure, $\mathrm{Pa}$

$P_{a}$ ambient pressure, $\mathrm{Pa}$

$\Delta P$ pressure difference between two components, $\mathrm{Pa}$

$\Delta T$ temperature difference of coolant and droplet, $\mathrm{Pa}$

$R$ radius of the melt droplet, $\mathrm{m}$

$R_{\delta}$ radius of the vapor bubble, $\mathrm{m}$

$V$ volume, $\mathrm{m}^{3}$

$m$ mass, $\mathrm{kg}$

$k$ coefficient of thermal conductivity, $\mathrm{W} /(\mathrm{m} \cdot \mathrm{K})$

$h \quad$ specific enthalpy, $\mathrm{J} / \mathrm{kg}$

$h_{f g}$ latent heat of evaporation, $\mathrm{J} / \mathrm{kg}$

$q$ heat flux, $\mathrm{W} / \mathrm{m}^{2}$

$U$ velocity, $\mathrm{m} / \mathrm{s}$

$c_{p}$ specific heat at constant pressure, $\mathrm{J} /(\mathrm{kg} \cdot \mathrm{K})$

$e$ specific internal energy, $\mathrm{J} /(\mathrm{kg} \cdot \mathrm{K})$

$Q$ thermal energy, $\mathrm{J}$

$L$ width of coolant around droplet, $\mathrm{m}$

$C_{A}$ constant related to the system

$C_{B}$ parameter related to droplet condition and acceleration

Th dimensionless number to describe the thermal condition of a system with a melt droplet and coolant

\section{Greeks}

$\rho$ density, $\mathrm{kg} / \mathrm{m}^{3}$

$\delta$ thickness of vapor film, $\mathrm{m}$

$\sigma$ surface tension, $\mathrm{N} / \mathrm{m}$

$\sigma_{r}$ Stefan-Boltzmann constant, $5.67 \times 10^{-8} \mathrm{~W} /\left(\mathrm{m}^{2} \cdot \mathrm{K}^{4}\right)$

$\alpha$ thermal diffusivity $\left(\mathrm{m}^{2} / \mathrm{s}\right)$

$\varepsilon$ emissivity

$\Gamma_{B E}$ fragmentation rate triggered by boiling effect, 1/s

\section{Subscripts}

$c$ : coolant

$v$ : vapor

$m$ : melt

frag: fragments 


\section{Development of Model}

\subsection{Acceleration Mechanism}

When a high temperature melt droplet is immersed into coolant liquid, a vapor film is formed to surround the droplet. At the beginning of the immersion, because the temperature of the droplet is over the minimum film boiling temperature (MFBT), the vapor film is stable. As the temperature of the droplet decreases to point $\mathrm{D}$, shown in the typical boiling curve in Fig.1, the vapor bubble becomes unstable and collapses toward the surface of the droplet. In this transition region, coolant periodically contacts the droplet surface. The transition can be very violent and continue until the nucleate boiling regime is attained at point $\mathrm{C}$. Due to the change of heat transfer mode from film boiling to nucleate boiling, the vapor bubble becomes unstable and a pressure pulse is generated at the vapor bubble collapse, which is exerted on the droplet surface and may induce the breakup of the droplet.

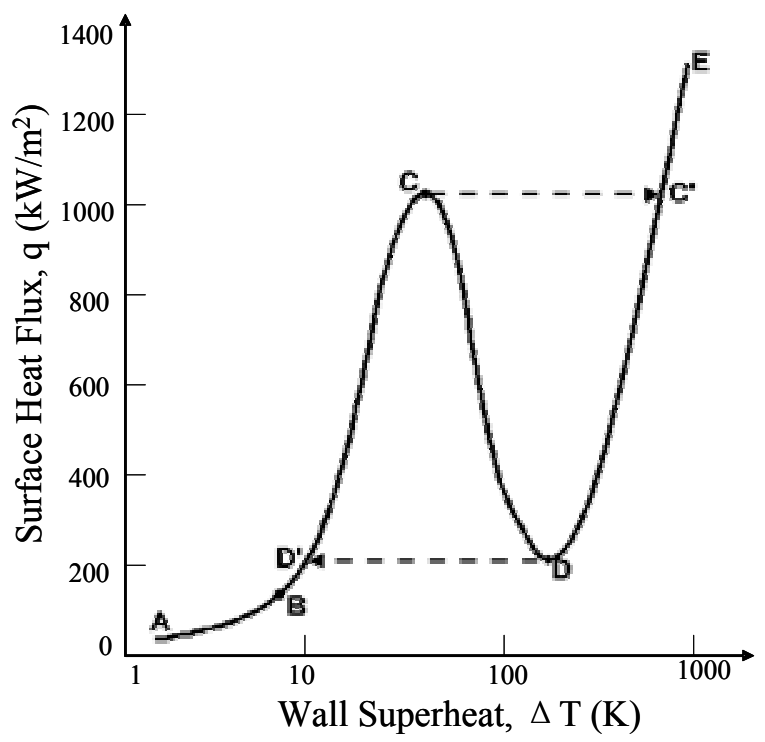

Fig.1 The typical boiling curve for saturated pool boiling of water at atmospheric pressure

\subsection{Vapor Bubble Collapse}

In this study, the vapor bubble collapse is modeled, which is similar to the method developed by Corradini ${ }^{(3)(4)}$. The vapor bubble collapse model due to heat transfer mode change is simulated by adding a heat flux term in the boundary condition of energy. The generation of the pressure during the vapor bubble collapse is related to the heat transfer change, the temperatures and the properties of the droplet and the coolant, and the system pressure.

The analytical model is shown in Fig.2. The dynamic vapor bubble collapse process is modeled by writing a momentum equation for vapor bubble dynamics and an energy equation for each region, as shown in Fig.2: melt droplet, vapor film and coolant and linking each region by the appropriate boundary conditions. The equilibrium is assumed on the interface between the vapor and coolant liquid. The integral approach is used in each region for the energy equations where the differential equation is integrated over the region and a temperature profile is assumed. 


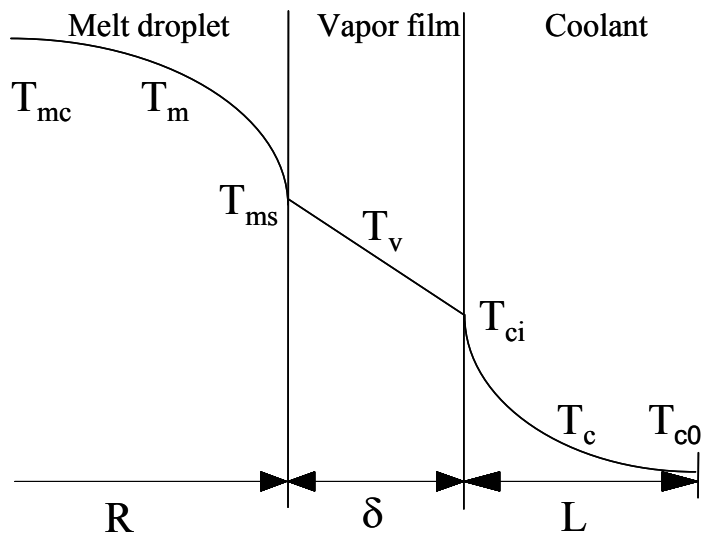

Fig. 2 The analytical model for vapor bubble collapse

The momentum equation for vapor bubble growth is modeled by the Rayleigh equation that neglects the effects of viscosity in a spherically symmetric system.

$$
\frac{d U}{d t}+\frac{3}{2} \frac{U^{2}}{R_{\delta}}=\frac{P_{v}-P_{c}}{\rho_{c} R_{\delta}}
$$

where $R_{\delta}$ is the radius of the vapor bubble which is sum of the radius of the droplet and the thickness of the vapor film,

$$
R_{\delta}=R+\delta .
$$

The ambient pressure during the growth of the vapor film is held constant.

Considering the evaporation of the coolant liquid on the interface, the velocity of the interface is given by

$$
\frac{d R_{\delta}}{d t}=U+\frac{d m_{v} / d t}{4 \pi R_{\delta}^{2} \rho_{c}},
$$

where $d m_{v} / d t$ is the evaporation (or condensation ) rate on the interface, which can be calculated by the energy equation of the vapor.

$$
\frac{d m_{v} e_{v}}{d t}=\frac{d Q}{d t}-P_{v} \frac{d V}{d t}+\frac{d m_{v}}{d t} h_{v},
$$

where the internal energy of the vapor is

$e_{v}=h_{v}-P_{v} / \rho_{v}$,

the volume of the vapor film is

$V=\frac{4}{3} \pi\left(R_{\delta}^{3}-R^{3}\right)$,

the enthalpy of vapor is

$h_{v}=c_{p} T_{v}$,

and 


$$
Q=\frac{2 k_{v}}{\delta}\left(T_{c i}-T_{v}\right)+\frac{2 k_{v}}{\delta}\left(T_{m s}-T_{v}\right) .
$$

The boundary conditions on the surface of the droplet and the interface of vapor and coolant can be written respectively as

$$
\left.k_{m} \frac{\partial T_{m}}{\partial r}\right|_{r=R}=\left.k_{v} \frac{\partial T_{v}}{\partial r}\right|_{r=R}+\varepsilon \sigma_{r}\left(T_{m s}^{4}-T_{c i}^{4}\right),
$$

and

$$
\left.k_{c} \frac{\partial T_{c}}{\partial r}\right|_{r=R+\delta}+\frac{d m_{v}}{d t} h_{f r a g}=\left.k_{v} \frac{\partial T_{v}}{\partial r}\right|_{r=R+\delta}+\varepsilon \sigma_{r}\left(T_{m s}^{4}-T_{c i}^{4}\right)+q .
$$

The last term of the right hand side of Eq.(10) is added to consider the boiling effect (local liquid-liquid contact). Considering the boiling curve for saturated pool boiling of water at atmospheric pressure, when the heat transfer mode changes from film boiling to nucleate boiling, the heat flux is increased about $8 \times 10^{5} \mathrm{~W} / \mathrm{m}^{2}$ (as shown in Fig.1, the transition from point $\mathrm{D}$ to point $\mathrm{C}$ ) which is set to the value of the last term in Eq.(10).

The temperature changes with time inside the droplet and coolant liquid near the vapor film can be written as

$$
\frac{\partial T_{m}}{\partial t}=\frac{\alpha_{m}}{r^{2}} \frac{\partial}{\partial r}\left(r^{2} \frac{\partial T_{m}}{\partial r}\right),
$$

and

$$
\frac{\partial T_{c}}{\partial t}+\frac{\partial r}{\partial t} \frac{\partial T_{c}}{\partial r}=\frac{\alpha_{c}}{r^{2}} \frac{\partial}{\partial r}\left(r^{2} \frac{\partial T_{c}}{\partial r}\right)
$$

where $\alpha_{m}=\frac{k_{m}}{\rho_{m} c_{p m}}$ and $\alpha_{c}=\frac{k_{c}}{\rho_{c} c_{p c}}$.

The pressure in the vapor film and the interfacial temperature are calculated by the equation of state of the coolant

$$
P_{v}=P_{v}\left(T_{v}, \rho_{v}\right),
$$

and

$$
T_{c i}=T_{\text {sat }}\left(P_{v}\right) .
$$

The temperature distributions in the $\mathrm{r}$ direction in the droplet and the coolant liquid are required to solve these equations. Here a quadratic temperature distribution in the droplet is assumed

$$
T_{m}=T_{m c}+\left(T_{m s}-T_{m c}\right)\left(1-\frac{x}{R}\right)^{2},
$$

where $x=R-r, x \in[0, R]$. The temperature distribution in the coolant liquid is assumed as

$$
\begin{aligned}
& T_{c}=T_{c 0}+\left(T_{c i}-T_{c 0}\right)\left(1-\frac{x}{L}\right)^{2}, \\
& \text { where } x=r-(R+\delta), x \in[0, L] .
\end{aligned}
$$


The vapor bubble collapse induced by the boiling effect (due to the change of the heat transfer mode from film boiling to nucleate boiling) can be modeled by the above equation system, which can be solved by the Runge-Kutta-Gill method.

An example is calculated, in which the heat flux change with $8 \times 10^{5} \mathrm{~W} / \mathrm{m}^{2}$ according to the boiling curve for saturated pool boiling of water at atmospheric pressure and the other initial conditions are taken from Nelson's experiment ${ }^{(2)}$. The calculation result shows that a pressure pulse is generated in the vapor bubble due to the change of the heat transfer mode, as show in Fig.3. The generated pressure pulse is up to a peak about $1.1 \mathrm{M} \mathrm{Pa}$ in a very short time (about $0.004 \mathrm{~ms}$ ).

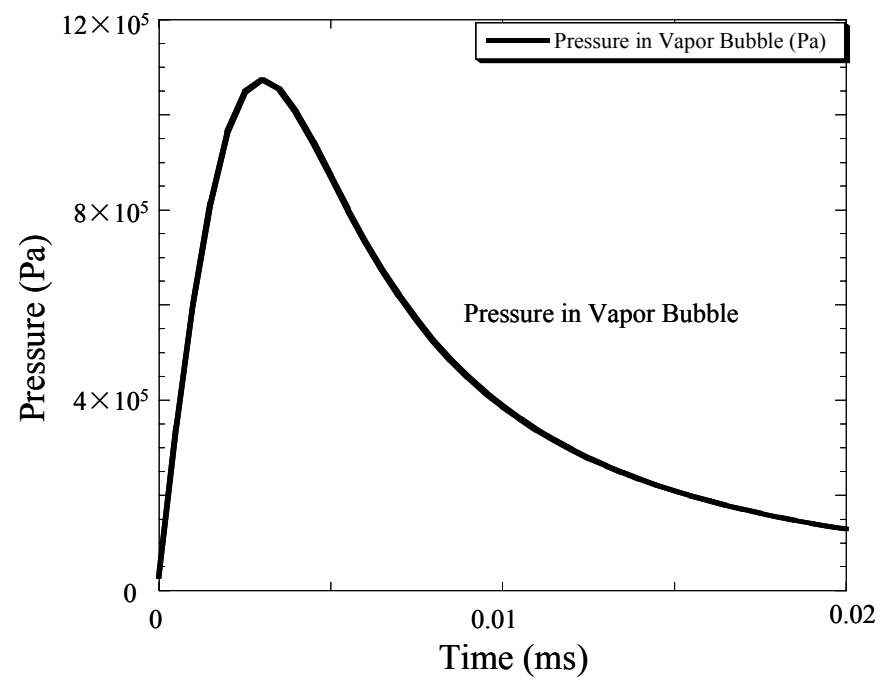

Fig. 3 The generated pressure in the vapor bubble due to heat transfer model change

\subsection{Fragmentation Model}

The generated pressure pulse in the vapor bubble can induce the instability of the droplet surface according to the instability analysis. The instability causes the fragmentation of the droplet. The development of fragmentation model is based on Ref.(5), in which the fragmentation rate can be written as

$$
\frac{1}{m_{0}} \frac{d m}{d t}=C_{A} C_{B} e^{C_{B} t},
$$

where $C_{A}$ is constant and $C_{B}$ is the parameter related to the droplet condition and the acceleration exerted on the droplet surface. The fragmented mass can be written as

$$
m_{\text {frag }}=\int_{0}^{t} \frac{1}{m_{0}} \frac{d m}{d t} d t .
$$

And the size of the fragments, which can be written as

$$
d_{\text {frag }}=\frac{d}{\left(\frac{\Delta P d}{2 \sigma}\right)^{0.25}}
$$

where $d_{\text {frag }}$ is the fragment size, $d$ is the diameter of the original droplet, $\sigma$ is the surface tension of the droplet and $\Delta P$ is pressure exerted on the droplet surface.

In this study of fragmentation triggered by the boiling effect, since the pressure pulse in the vapor bubble is related to the evaporation rate difference between the stable vapor film 
boiling and nucleate boiling, which is related to the difference of the heat transfer to the interface of the vapor/coolant liquid, the temperature of droplet and coolant, radius of the droplet, and the properties of the materials involved. To use the developed formula Eq.(17) as a tool for numerical experiments, the averaged fragmentation rate, $\Gamma_{B E}(1 / \mathrm{sec})$, of a melt droplet triggered by boiling effect, is fitted as the following formula, described by thermal parameters

$$
\Gamma_{B E}=56 T h^{-0.25}\left(\frac{q}{R h_{f g} \rho_{c}}\right),
$$

where $\Gamma_{B E}$ is the fragmentation rate, defined as normalized fragmented mass of a droplet (fragmented mass is divided by initial droplet's mass) per second, $T h=\left(\frac{k_{m} k_{c} \Delta T^{2}}{h_{f g}^{2} R^{2} \rho_{c} P_{a}}\right)$, which is a dimensionless number to describe the thermal conditions of the system (including melt droplet and coolant), and $q$ is the heat flux change due to the mode change from film boiling to nucleate boiling. This correlation is fitted within the range of: $q$, from $5 \times 10^{5}$ to $1 \times 10^{6} \mathrm{~W} / \mathrm{m}^{2} ; T_{m}$, from 673 to $1073 \mathrm{~K} ; R$, from 0.001 to $0.01 \mathrm{~m} ; P_{a}$, from $10^{5}$ to $10^{6} \mathrm{~Pa}$; and $T_{c}$, from 283 to $353 \mathrm{~K}$.

By using the vapor bubble collapse model as a tool for numerical experiments, the averaged pressure buildup due to boiling effect is fitted as

$$
\Delta P=3.7 \times 10^{3} T h^{-0.61}\left(\frac{q}{h_{f g} \rho_{c}}\right)^{2} \rho_{c} .
$$

The averaged fragments' size can be also estimated by Eq.(19) with an averaged pressure buildup in vapor film during vapor bubble collapse.

The proposed fragmentation rate increases with the increase of the ambient pressure, but it does not mean that high ambient pressure induces high probability of the occurrence of the fragmentation, which conflict with the fact observed in experiments. Since the probability of the occurrence of the triggering event is not studied, in the fitness of the proposed correlation, the triggering condition is assumed that the surface temperature of the droplet is reached to the MFBT of coolant. Then the unstable heat transfer mode causes the high pressure pulse in the vapor bubble. When the ambient pressure is increased, the vapor film becomes more stable, which reduces the probability of triggering, which will be considered in the simulation tool.

\section{Verification of the Proposed Thermal Fragmentation Model}

\subsection{Verification with experiment}

The size of debris in the experiment of Ref.(6) is selected to verify the proposed fragmentation model triggered by boiling effect. The initial temperature of the melt droplet is about $1000 \mathrm{~K}$. The prediction of fragment sizes by Eq.(19) is $0.54 \mathrm{~mm}$, which is near to the experimental data of 0.1 to $0.5 \mathrm{~mm}$ estimated from the photos of the debris, indirectly verifying the proposed thermal model.

Although the proposed fragmentation model triggered by boiling effect is introduced into computer codes, due to lack of the multi-droplet experiment, in which the 
fragmentation is triggered by the boiling effect, the proposed thermal fragmentation model is not verified by the simulation of multi-droplet experiment. And, under the condition of the reactor cases, the thermal fragmentation triggered by the boiling effect may not be the dominant fragmentation mechanism.

\subsection{Sample Calculation}

An example is calculated by using the proposed model. The initial condition is that a melt droplet with radius of $1 \mathrm{~cm}$ and melting temperature of $400 \mathrm{~K}$ is immersed into water with temperature of $300 \mathrm{~K}$. The initial temperature of melt droplet is $800 \mathrm{~K}$, which is higher than the spontaneous nucleation temperature. Since the melting point is less than MFBT of water, after the temperature of melt droplet decreased below the MFBT, the droplet hasn't been solidified. During this period, the boiling effect dominates the fragmentation of the droplet. When the melting point is less than the MFBT, in order to estimate the pressure generation in the vapor bubble due to the change of the heat transfer mode from film boiling to nucleate boiling, it is assumed that the heat fluxes at the MFBT and at nucleate boiling are $2 \times 10^{5} \mathrm{~W} / \mathrm{m}^{2}$ and $10^{6} \mathrm{~W} / \mathrm{m}^{2}$ respectively. By using the developed bubble collapse model above, the fragmented mass induced by the boiling effect is calculated by Eq.(18), as shown in Fig.4.

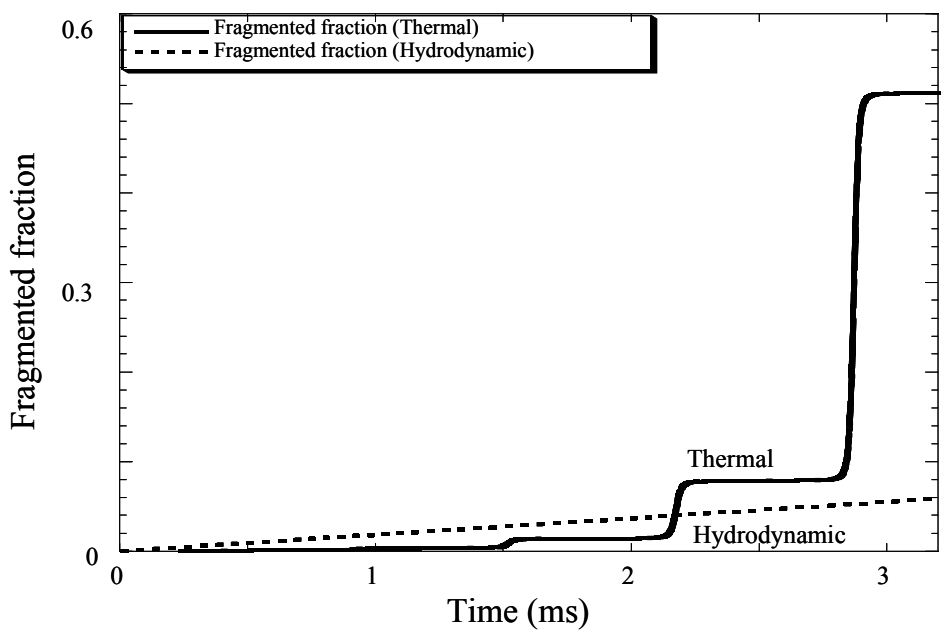

Fig.4 Fragmented mass induced by external pressure pulse applying on the melt droplet

After the relative velocity of the melt droplet to coolant is assumed to be $5 \mathrm{~m} / \mathrm{s}$, usual velocity in the single droplet experiment, the calculated results show that the thermal fragmentation model given by the proposed model is much larger than that given by hydrodynamic model in Ref.(7).

\section{Conclusions}

In this study, based on the understanding of the thermal fragmentation mechanisms, in which a pressure pulse is generated and exerted on the melt droplet, for developing the fragmentation model, the vapor bubble collapse model is developed to estimate the generated pressure. And then, a thermal fragmentation model is proposed for the triggering events of boiling effect. For the convenient application of the model to the simulation tools, simplified correlations of the thermal fragmentation model are proposed, based on the fragmentation mechanism. The proposed model is indirectly verified by comparing the prediction of the fragment size with the experimental data collected after these experiments.

The thermal fragmentation mechanisms are related to the triggering events. Under 
experimental or reactor conditions, the triggering event is determined by the thermal conditions and other mechanical conditions in the system. Those conditions are not all considered in this study, in which the instability is proposed to be the thermal fragmentation mechanism. In the future the more triggering events should be studied. The proposed thermal fragmentation models should be verified by simulating more experiments with different thermal conditions.

\section{Acknowledgment}

Work supported by National Natural Science Foundation of China under Grant No. 50576050 and the NSFC-RFBR Joint Program under Grant No.50711120190

\section{References}

(1) Corradini, M. L., Kim, B.J., Oh, M.D., Vapor explosion in light water reactor: a review of theory and modeling, Progress in Nuclear Energy, Vol.22, No.1(1988), pp. 1-117.

(2) Nelson, L. S., Duda, P.M., Steam explosion experiments with single drops of iron oxide melted with a $\mathrm{CO}_{2}$ laser part II. parametric studies, NUREG/CR-2718, SAND82-1105, 1985.

(3) Corradini, M. L., Phenomenological modeling of the triggering phase of small-scale steam explosion experiments, Nuclear Science and Engineering, Vol.78(1981), pp. 154-170.

(4) Corradini, M. L., Modeling film boiling destabilization due to a pressure shock arrival, Nuclear Science and Engineering, Vol.84, No.3(1983), pp. 196-205.

(5) Cao, X., Tobita, Y., Kondo, S., A thermal fragmentation model induced by surface solidification, Journal of Nuclear Science and Technology, Vol.39, No.6(2002), pp. 628-636.

(6) Mishima, K., Hibiki, T., Satio, Y., Visualization study of molten metal-water interaction by using neutron radiography, Nuclear Engineering and Design, Vol.189, No.1(1999), pp. 391-403.

(7) Sawada, T., Ninokata, H., Validation of a computational model for the evaluation of fuel-coolant interaction under severe accidental condition in fast breeder reactors, Annals of Nuclear Energy, Vol.25, No.18 (1998), pp.1543-1552. 